\title{
Predictive Variables Affecting Transfusion Requirements in Orthotopic Liver Transplantation
}

\author{
T. Araújo, A. Cordeiro, P. Proença, R. Perdigoto, A. Martins, and E. Barroso
}

\begin{abstract}
Introduction and aims. Adult orthotopic liver transplantation (OLT) is associated with considerable blood product requirements. The aim of this study was to assess the ability of preoperative information to predict intraoperative red blood cell (RBC) transfusion requirements among adult liver recipients.

Methods. Preoperative variables with previously demonstrated relationships to intraoperative RBC transfusion were identified from the literature: sex, age, pathology, prothrombin time $(\mathrm{PT})$, factor $\mathrm{V}$, hemoglobin $(\mathrm{Hb})$, and platelet count (plt). These variables were then retrospectively collected from 758 consecutive adult patients undergoing OLT from 1997 to 2007. Relationships between these variables and intraoperative blood transfusion requirements were examined by both univariate analysis and multiple linear regression analysis.

Results. Univariate analysis confirmed significant associations between RBC transfusion and PT, factor V, Hb, Plt, pathology, and age $(P$ values all $<.001)$. However, stepwise backward multivariate analysis excluded variables Plt and factor $\mathrm{V}$ from the multiple regression linear model. The variables included in the final predictive model were $\mathrm{PT}, \mathrm{Hb}$, age, and pathology. Patients suffering from liver carcinoma required more blood products than those suffering from other pathologies. Yet, the overall predictive power of the final model was limited $\left(R^{2}=.308\right.$; adjusted $\left.R^{2}=.30\right)$.

Conclusion. Preoperative variables have limited predictive power for intraoperative $\mathrm{RBC}$ transfusion requirements even when significant statistical associations exist, identifying only a small portion of the observed total transfusion variability. Preoperative PT, $\mathrm{Hb}$, age, and liver pathology seem to be the most significant predictive factors but other factors like severity of liver disease, surgical technique, medical experience in liver transplantation, and other noncontrollable human variables may play important roles to determine the final transfusion requirements.
\end{abstract}

$\mathbf{T}^{\mathrm{H}}$ HE FIRST HUMAN LIVER TRANSPLANTATION was performed by Starzl et al in 1963. Unfortunately, this first patient along with the next four died from bleeding during the procedure, exemplifying what would become one of the most important barriers in OLT in the following years.

Improvements in surgical techniques, anesthesia management, and organ preservation have led to an overall significant reduction in intraoperative blood loss among adult liver recipients. ${ }^{1}$ Yet the possibility of major intraoperative blood loss and the subsequent transfusion of large amounts of blood products still pose a real concern. After revising the transfusional chart of 758 consecutive transplanted adult patients we verified that $17.9 \%$ of OLT procedures required more than 11 red blood cell (RBC) units. The aim of this study was to assess the ability of preoperative variables to predict bleeding and intraoperative blood transfusion requirements among adult liver recipients.

From the Serviço de Imunohemoterapia, Hospital Curry Cabral (T.A., A.C.), Lisboa, Portugal; Instituto Superior de Economia e Gestão (P.P.), Lisboa, Portugal; and Centro Hepato-Bilio-Pancreático, Hospital Curry Cabral (R.P., A.M., E.B.), Lisboa, Portugal.

Address reprint requests to Teresa Araújo, Hospital Curry Cabral, Rua da Beneficência, No. 8, 1069-166 Lisboa, Portugal. E-mail: mtaraujo@hccabral.min-saude.pt

(C) 2010 by Elsevier Inc. All rights reserved. 360 Park Avenue South, New York, NY 10010-1710 


\section{METHODS}

Medical records from 758 consecutive adult patients who underwent OLT between January 1997 and December 2007 in our center were included in this retrospective study. There were 266 female and 492 male patients. Mean age for the entire group was 44 (13-70) years. The pathologies were distributed in six categories: familial amyloidotic polyneuropathy $(\mathrm{n}=215)$; primary alcool $(n=99)$; primary virus $(n=113)$; primary tumor $(n=122)$; other pathologies including Wilson disease and primary biliary cirrhosis $(\mathrm{n}=126)$ and retransplantations $(\mathrm{n}=83)$.

Preoperative variables with a previously demonstrated relationship to intraoperative blood transfusion were identified from the literature ${ }^{2,3}$ : sex, age, pathology, prothrombin time (PT), factor V, hemoglobin $(\mathrm{Hb})$, and platelet count (plt). Other characteristics suggested to be of interest by clinical experience,- - patient's previous upper abdominal surgery and donor factors such as poor early graft function and liver split transplants-were analyzed. After assessing their low incidence in our patient population (Table 1), they were not included in this study. We also did not include the variables of Child classification and surgical team experience. Relationships between the variables and intraoperative blood transfusion requirements were examined by both univariate and multivariate analyses as well as multiple linear regression analysis.

\section{RESULTS}

Univariate analysis confirmed significant associations between RBC transfused and the preoperative variables: PT, factor $\mathrm{V}, \mathrm{Hb}$, Plt, pathology, and age $(P$ values all $<.001)$. Only the sex variable was marginally significant $(P=.051)$. However, stepwise backward multivariate analysis excluded Plt and factor $\mathrm{V}$ from the multiple regression linear model (criterion: probability of $\mathrm{F} » 0.1$ ). The variables included in the final predictive model were: PT [Beta $(\mathrm{B})-0.034$; standard error (SE) 0.007], $\mathrm{Hb}$ [B - 0.656; SE 0.066], age [B 0.044; SE 0.012], and pathology. Patients suffering from liver carcinoma also proved to require more blood products than those suffering from other pathologies. The model obtained was: $\mathrm{RBC}=8.841-0.043 \mathrm{PT}-0.249 \mathrm{Hg}+0.060$ age + constant (constant: PAF +0 ; Alcool +1.773 ; viral + 1.861 ; carcinoma +2.5 ; other pathology +1.319 retransplantation +1.647$)$. However, the overall predictive power of the final model was limited $\left(R^{2}=.308\right.$; adjusted $\left.R^{2}=.30\right)$.

\section{DISCUSSION}

Profound, complex coagulation disorders are encountered during OLT: preoperative coagulation disorders related to the disease and hemostatic changes related to the procedure, which commonly increase intraoperative bleeding during OLT.

Table 1. Other Clinical Characteristics of the 758 OLT Patients

\begin{tabular}{lc}
\hline \multicolumn{1}{c}{ Variable } & $n(\%)$ \\
\hline Previous upper abdominal surgery & $16(2.1)$ \\
Poor early graft function & $10(1.3)$ \\
Split liver transplants & $14(1.8)$
\end{tabular}

OLT, orthotopic liver transplantation.
Despite recent progress in anesthesiology and surgical care, hemorrhage remains a problem during OLT. The ability to predict intraoperative bleeding would be of great value in the management of the surgical procedure, as it would allow the selection of patients more likely to benefit from blood-saving strategies including the utilization of antifibrinolytic agents like aprotinin or the prophylactic application of rFVIIa, ${ }^{4}$ which decreases blood transfusion requirements only when surgery is associated with significant blood loss.

Several groups have attempted to identify patients at high risk of bleeding and to define preoperative factors related to increased intraoperative bleeding. The results have not been homogenous as patient selection, surgical conditions, and intraoperative management vary from center to center. ${ }^{5}$ With this study, we tried to identify preoperative variables with the ability to predict bleeding and intraoperative blood transfusion requirements for our adult OLT patients. We focused essentially on preoperative hematologic and coagulation tests.

Statistical analysis confirmed significant associations between the variables included in the study (and those previously identified in the literature) and transfused RBC: PT, factor $\mathrm{V}, \mathrm{Hb}$, Plt, pathology, and age. Multiple linear regression analysis excluded factor $\mathrm{V}$ and Plt from the final predictive model. The predictive roles of PT and $\mathrm{Hgb}$ observed in our study were expected, but the exclusion of Plt was not, as its number is usually correlated with bleeding identified in other studies. ${ }^{6}$

In conclusion, the aim of the present prospective survey was to evaluate whether the intraoperative RBC transfusion could be anticipated from preoperative clinical and laboratory values in our patient population. Statistical analysis yielded a predictive mathematical model that included PT, $\mathrm{Hb}$, age, and pathology. Yet the predictive power of the final model was limited. We concluded that the preoperative variables studied herein only explain in part the variability. Although these variables cannot predict intraoperative blood loss and $\mathrm{RBC}$ requirements on an individual basis because of their limited sensitivity, they remain statistically significant risk factors that are helpful to identify patients at greater risk of requiring transfusions.

\section{REFERENCES}

1. Boer MT, Molenaar IQ, Hendriks HG, et al: Minimizing blood loss in liver transplantation: progress through research and evolution of techniques. Dig Surg 22:265, 2005

2. Porte RJ: Coagulation and fibrinolysis in orthotopic liver transplantation: current views and insights. Semin Thromb Hemost 19:191, 1993

3. Ozier J, Pessione F, Samain E, et al: Institutional variability in transfusion practice for liver transplantation. Anesth Analg 97:671, 2003

4. Porte RJ, Caldwell SH: The role of recombinant factor VIIa in liver transplantation. Liver Transpl 8:872, 2005

5. Steib A, Frey G, Lehmann C, et al: Intraoperative blood losses and transfusion requirements during adult liver transplantation remain difficult to predict. Can J Anesth 48:1075, 2001

6. Massicotte L, Sassine MP, Lenis S, et al: Transfusion predictors in liver transplant. Anesth Analg 98:1245, 2004 